\title{
Experimental Overview on Heavy Flavor Production in Heavy lon Collisions.
}

\author{
Cesar L. da Silva ${ }^{1, \star}$ \\ ${ }^{1}$ Los Alamos National Lab - USA
}

\begin{abstract}
The use of probes containing heavy quarks is one of the pillars for the study of medium formed in high energy nuclear collisions. The conceptual ideas formulated more than two decades ago, such as quark mass hierarchy of the energy that the probe lose in the media and color screening of bound heavy quarkonia states, have being challenged by the measurements performed at RHIC and LHC. A summary of the most recent experimental observations involving charm and bottom quarks in pp, pA, and AA collisions from collisions energies extending from $\sqrt{s_{N N}}=200 \mathrm{GeV}$ to $8 \mathrm{TeV}$ is presented. This manuscript also discuss possibilities of new measurements which can be at reach with increased statistics and detector upgrades.
\end{abstract}

\section{Open heavy flavor: Charm and Bottom Quarks}

The characterization of media formed in high energy collisions, such as the Quark-Gluon-Plasma (QGP), relies on the use of a probe which is produced and do not shower in the vacuum before the medium formation. Probes with different rest masses are required, and measurements may extend in a broad transverse momentum range. Charm(bottom)-quarks have formation times of $0.07(0.01) \mathrm{fm} / c$, much earlier than any prediction for QGP formation. The number of charm and bottom quarks, once created by a hard scattering, is preserved when crossing the medium [1-3]. Two main steps are needed to understand the heavy flavor (HF) probe before use it to make QGP tomography: 1) understand the production mechanisms and cross-sections in $p+p$ collisions; and 2) understand how the HF sources are affected when the nucleon is in a nucleus.

Heavy flavor cross-section measurements reached a level of precision much better than the stateof-the-art perturbative Quantum Cromodynamics calculations, some examples can be found in [4-6]. Heavy flavor is mainly produced from gluons at RHIC and LHC. According to PYTHIA simulations of hard processes [7], heavy-quark pair creation and next-to-leading-order flavor excitation are the dominant production sources at RHIC energies $\left(\sqrt{s_{N N}}=200 \mathrm{GeV}\right)$. Figure 1 shows how these processes produce different azimuthal correlations of muons from $c \bar{c}$ and $b \bar{b}$ pairs. As the energy increases at Tevatron and LHC, the gluon splitting contribution becomes dominant [8]. This is a fundamental difference between HF production at RHIC and LHC.

Initial state effects must be considered for HF production in collisions involving nucleus. Data from $d+\mathrm{Au}$ collisions taken at RHIC shows a significant enhancement of HF yield relative to $p+p$

^e-mail: cesar_luiz@lanl.gov 

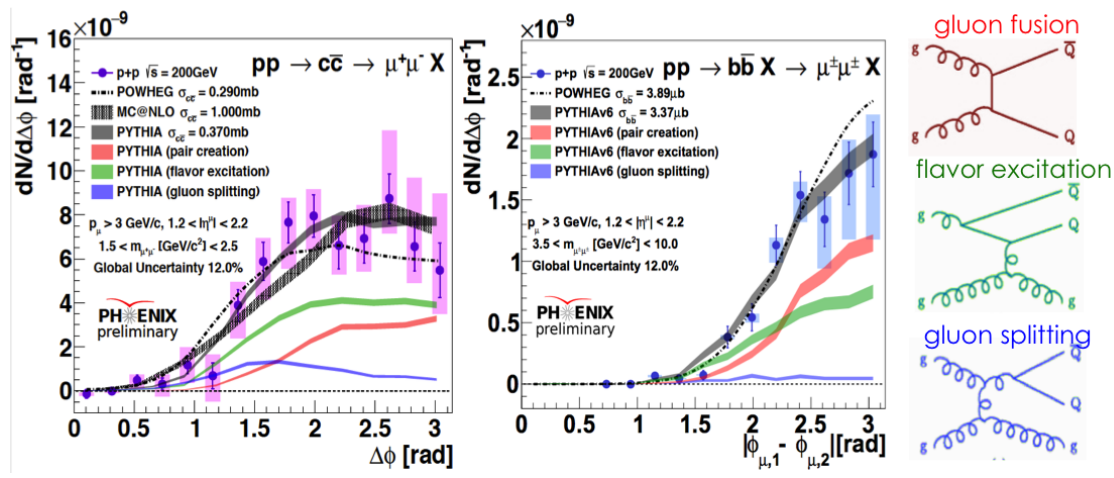

Figure 1: Azimuthal correlation of charm and bottom pairs from PHENIX at RHIC described by different heavy flavor production processes obtained from PYTHIA [7].

collisions at mid- and backward-rapidities $[9,10]$, corresponding to moderated to large fractional momentum $x$. Measurements from $p+\mathrm{Pb}$ collisions at LHC typically show no significant yield modification at mid- and backward rapidities [11, 12], however ALICE also reports a significant enhancement for the most central collisions. One explanation for these enhancements is incoherent multiple scattering of the initial gluon in the nucleus matter [13] and Cronin $p_{T}$ broadening [14]. A significant $\mathrm{HF}$ suppression is observed at the forward direction of $p+\mathrm{Pb}$ collisions by $\mathrm{LHCb}$ [12], covering partons with small fractional momentum $x$. This suppression is attributed to shadowing [15], energy loss before the hard scattering [16], and gluon saturation [17, 18].

Heavy quarks lose energy $\Delta E$ when crossing the QGP medium through collisional scattering and gluon radiation [19]. Between 80-70\% of high $p_{T}$ mesons are consistently suppressed in central heavy ion collisions at RHIC and LHC, regardless its mass or collision energy [20]. Data from STAR (Dmesons) [1], PHENIX $(c+b \rightarrow e)$ [21], ALICE(D-mesons) [22] and CMS(D-meson, B-meson and $\mathrm{B} \rightarrow \mathrm{J} / \psi)[23,24]$ indicate that quark mass dependency on the energy loss in the QGP $(\Delta E(u, d)>$ $\Delta E(c)>\Delta E(b))$, caused by the suppression of gluon radiation in a cone $\Delta \theta<m_{q} / E$ [25] where $m_{q}$ is the quark mass and $E$ its energy, is apparently relevant only for $p_{T} \lesssim m_{q}$. Figure 2 compares the nuclear modification fraction $R_{A A}$ of charm and bottom decays at RHIC using unfolding of electron decays on the left panel; $D$-mesons, $B$-meson and $B \rightarrow J / \psi$ at LHC are shown on the right panel. It is still not evident the maximum $p_{T}$ where the meson suppression has a flavor dependency. Extra caution is also needed to interpret the data sets to account for the presence of initial state effects, such as the yield enhancements observed in $d+$ Au collisions at RHIC.

Direct access to the initial heavy quark can be done with jets. CMS reported that $b$-jets with $p_{T}>80 \mathrm{GeV} / c$ have the same suppression as inclusive jets dominated by light quarks and gluons [26]. One possible explanation for the similar behavior between light and heavy quarks in the QGP medium is that heavy quarks are mainly produced in gluon splittings at LHC. Heavy quarks from these processes behave like gluons crossing the medium [27]. A preliminary study performed by CMS selects back-to-back $b$-jet pairs, minimizing the contribution of gluon splittings. The modification of the momentum imbalance between the $b$-jet pair in $\mathrm{Pb}+\mathrm{Pb}$ collisions, relative to $p+p$ collisions, still shows the same behavior as inclusive jets.

The second (elliptic flow) and third (triangular flow) Fourier components $v_{n}$ of D-meson azimuthal asymmetry relative to the event reaction plane, measured at RHIC [28] and LHC [29, 30], indicate that charm quarks are strongly coupled to the medium with a significant diffusion coefficient. However, 

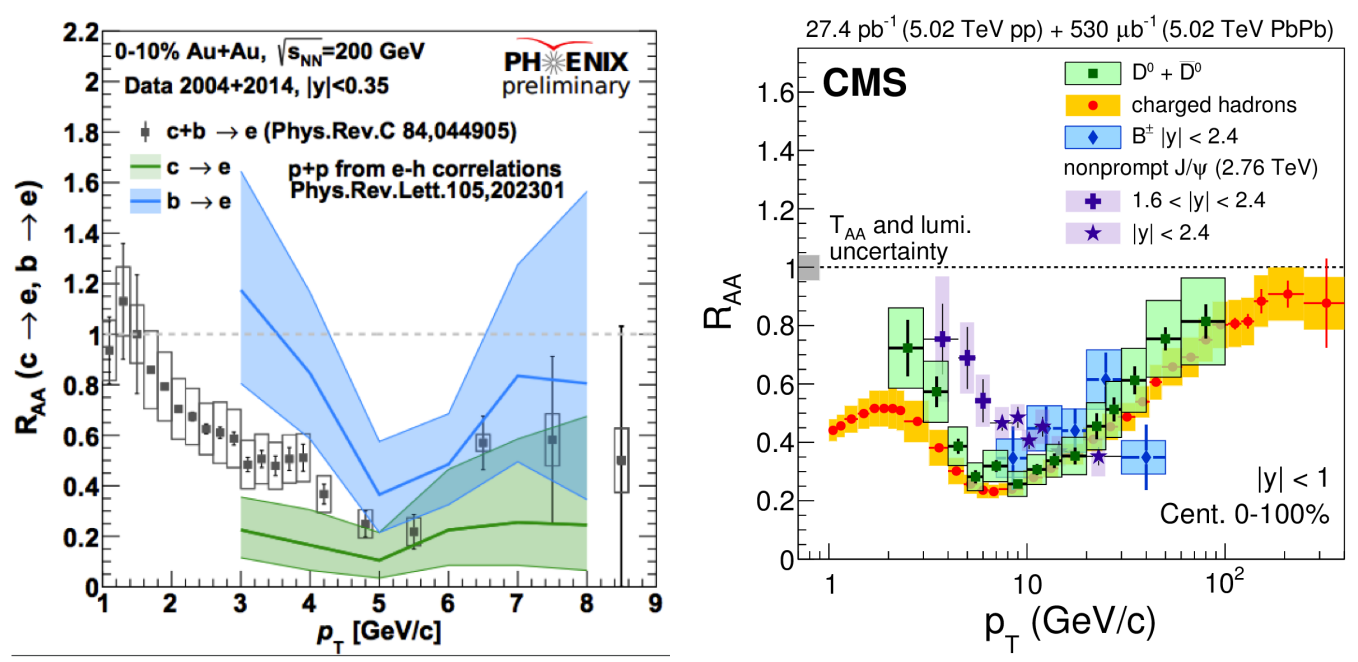

Figure 2: Nuclear modification factor of charm and bottom quarks versus transverse momentum measured by PHENIX at RHIC (left) and CMS at LHC (right).

significant $v_{2}$ have also been seen in many small system measurements, such as $d+\mathrm{Au}$ and $p+\mathrm{Pb}$ collisions.

\section{Quarkonia}
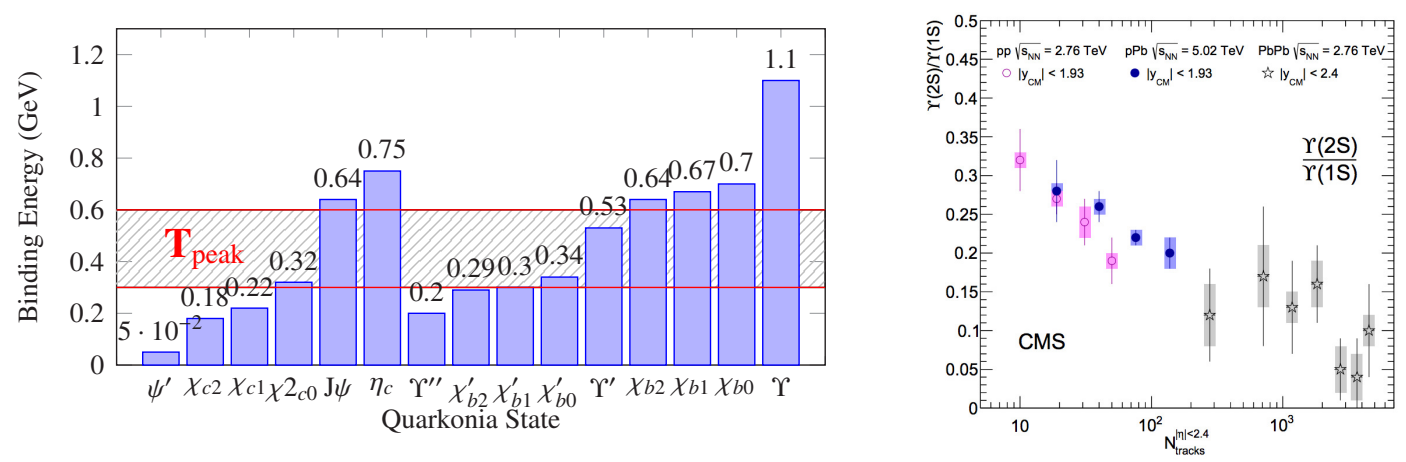

Figure 3: (left) Binding energy of several quarkonium states in vacuum [31] along with an estimation for the quark-gluon plasma peak temperature at RHIC [32] as hashed area. (right) Relative $\Upsilon(2 \mathrm{~S}) / \Upsilon(1 \mathrm{~S})$ yields versus particle multiplicity in $p+p, p+\mathrm{Pb}$ and $\mathrm{Pb}+\mathrm{Pb}$ collisions measured by $\mathrm{CMS}$ [33].

Quarkonia states have a broad range of binding energies (Fig.3-left). The suppression (or not) of these different states depends on the local free energy of the medium. Recent experimental studies started to emphasize on the relative nuclear modifications between different quarkonia states, cancel- 
ing out initial state effects. Examples are the suppression of the $\psi(2 \mathrm{~S})$ relative to $\mathrm{J} / \psi$ in small system collisions at RHIC and LHC [34-36].

According to charm cross section measurements, each central Au+Au collision at RHIC produce around $20 c \bar{c}$ pairs. In LHC this number is ten times larger. There is a significant probability for these charm quarks to recombine and form a new source of bound state charmonia in heavy ion collisions. PHENIX measurements indicate that $\mathrm{J} / \psi$ is less suppressed at mid-rapidity, where the number of $c \bar{c}$ pairs is the largest, compared to large rapidity [37]. Measurements in ALICE shows a nuclear modification similar to the large rapidity reported by PHENIX only at high $p_{T}$. The suppression is largely reduced towards low $p_{T}$ [38]. These two observations can be evidences of charm recombination. A significant $\mathrm{J} / \psi$ elliptic flow $v_{2}$ is observed by ALICE [39], suggesting that the charm flow is transfered to $\mathrm{J} / \psi$ produced from coalescence.

Because of the much smaller number of $b \bar{b}$ pairs available per event, bottomonium states are expected to have small contribution from bottom recombination, even at LHC. A sequential suppression of $\Upsilon$ states has been observed by CMS in several collision systems. A pattern emerges when looking at Figure 3-right, which shows the ratio between $\Upsilon(2 S) / \Upsilon(1 S)$ yields versus particle activity in the event at the same rapidity range of the measured $\Upsilon$ yields. The relative suppression of the weaker bounded $2 \mathrm{~S}$ state increases with local particle density, regardless the collision system $(p+p, p+\mathrm{Pb}$ or $\mathrm{Pb}+\mathrm{Pb}$ ) [33]. This observation challenges the long standing picture of color screening of quarkonia states only in QGP medium [40].

\section{Conclusions, Future Studies and Facilities}

More than 15 years of data from RHIC and LHC shows that the QGP medium formed in high-energy nuclear collisions is opaque to high $p_{T}$ hard probes, and the energy the hig- $p_{T}$ probe lose in the medium does not depends on its constituent quark mass. The transition where the quark mass matters for the energy that the probe lose in the medium is still to be determined. Caution is also needed to correctly account for nuclear effects before the probe cross the medium. More studies are also needed for a conclusion on the diffusion coefficients obtained from charm flow measurements. The questions raised above drive the interest on low- $p_{T}$ heavy quark measurements in the near future. New results will emerge from the data being analyzed from the recent high luminosity RHIC runs and LHC.

Higher harmonics $v_{n}>2$ are starting to be available for D-mesons. Preliminary results on Bmeson $v_{2}$ started to be available by CMS collaboration. The set of HF $v_{n}$ measurements will help to elucidate how heavy quarks couple with the medium, and indicate how geometrical aspects of the collisions affect these results. Heavy quark correlations using semi-leptonics decays, mesons or jets have also a potential to select different production processes and even quantify the magnitude of collisional and radiative energy loss in QGP. Several detector upgrades are expected in LHC accessing a new level of precision in HF measurement. The sPHENIX upgrade at RHIC [41] will dedicates to jets and HF measurements, including D-, B-mesons and b-jets.

We are seeing a new wave of measurements now focusing in the excited state charmonia and bottomonium. Yield modifications relative to ground states reduces misinterpretations caused by initial state effects. Recent measurements of $\Upsilon$ indicate that states with weaker binding have a suppression relative to ground states which mainly depends on the local particle densities, regardless the collision system. New $\Upsilon$ measurements are expected from RHIC with different particle densities. Measurement of radiative decays of $\chi_{C}$ states is a promise from LHCb, which would greatly expands the understanding of charmonium breaking in heavy ion collisions. 


\section{References}

[1] L. Adamczyk et al. (STAR Collaboration), Phys. Rev. Lett. 113, 142301 (2014)

[2] A. Adare et al. (PHENIX), Phys. Rev. Lett. 98, 172301 (2007)

[3] C. Aidala et al. (PHENIX Collaboration), Phys. Rev. C 96, 064901 (2017)

[4] L. Adamczyk et al. (STAR Collaboration), Phys. Rev. D 86, 072013 (2012)

[5] D. Acosta et al. (CDF), Phys. Rev. Lett. 91, 241804 (2003)

[6] S. Acharya et al. (ALICE), Eur. Phys. J. C77, 550 (2017)

[7] T. Sjöstrand, S. Ask, J.R. Christiansen, R. Corke, N. Desai, P. Ilten, S. Mrenna, S. Prestel, C.O. Rasmussen, P.Z. Skands, Computer Physics Communications 191, 159 (2015)

[8] V. Khachatryan et al. (CMS), JHEP 03, 136 (2011)

[9] A. Adare et al. (PHENIX), Phys. Rev. Lett. 112, 252301 (2014)

[10] A. Adare et al. (PHENIX), Phys. Rev. Lett. 109, 242301 (2012)

[11] ALICE-PUBLIC-2017-008 (2017)

[12] R. Aaij et al. (LHCb), JHEP 10, 090 (2017)

[13] Z.B. Kang, I. Vitev, E. Wang, H. Xing, C. Zhang, Phys. Lett. B740, 23 (2015)

[14] I. Vitev, Phys. Lett. B562, 36 (2003)

[15] N. Armesto, J. Phys. G32, R367 (2006)

[16] R. Neufeld, I. Vitev, B.W. Zhang, Physics Letters B 704, 590 (2011)

[17] L.D. McLerran, R. Venugopalan, Phys. Rev. D49, 3352 (1994)

[18] F. Gelis, E. Iancu, J. Jalilian-Marian, R. Venugopalan, Ann. Rev. Nucl. Part. Sci. 60, 463 (2010)

[19] J. Uphoff, O. Fochler, Z. Xu, C. Greiner, J. Phys. G42, 115106 (2015)

[20] A.M. Sirunyan et al. (CMS Collaboration), Phys. Rev. Lett. 119, 152301 (2017)

[21] A. Adare et al. (PHENIX), Phys. Rev. C93, 034904 (2016)

[22] J. Adam et al. (ALICE), JHEP 03, 081 (2016)

[23] A.M. Sirunyan et al. (CMS) (2017), nucl-ex: 1708.04962

[24] V. Khachatryan et al. (CMS), Eur. Phys. J. C77, 252 (2017)

[25] Y. Dokshitzer, D. Kharzeev, Physics Letters B 519, 199 (2001)

[26] S. Chatrchyan et al. (CMS Collaboration), Phys. Rev. Lett. 113, 132301 (2014)

[27] J. Huang, Z.B. Kang, I. Vitev, Phys. Lett. B726, 251 (2013)

[28] L. Adamczyk et al. (STAR), Phys. Rev. Lett. 118, 212301 (2017)

[29] S. Acharya et al. (ALICE) (2017), nucl-ex: 1707.01005

[30] A.M. Sirunyan et al. (CMS) (2017), nucl-ex: 1708.03497

[31] H. Satz, J. Phys. G 32, R25 (2006)

[32] A. Adare et al. (PHENIX Collaboration), Phys. Rev. C 81, 034911 (2010)

[33] S. Chatrchyan et al. (CMS), JHEP 04, 103 (2014)

[34] A. Adare et al. (PHENIX Collaboration), Phys. Rev. C 95, 034904 (2017)

[35] B.B. Abelev et al. (ALICE), JHEP 02, 073 (2014)

[36] R. Aaij et al. (LHCb), JHEP 03, 133 (2016)

[37] A. Adare et al. (PHENIX), Phys. Rev. Lett. 98, 232301 (2007)

[38] J. Adam et al. (ALICE), Phys. Lett. B766, 212 (2017)

[39] E. Abbas et al. (ALICE), Phys. Rev. Lett. 111, 162301 (2013)

[40] F. Karsch, M.T. Mehr, H. Satz, Z. Phys. C 37, 617 (1987)

[41] A. Adare et al. (2015), nucl-ex: 1501.06197 